pollen have been identified, predominantly herbiage (Graminiae, Cyberaceae, Caryophyllaceae, Ranunculus, Rosaceae and Compositae) amounting to $80.9 \%$ of the whole, with smaller quantities of spores $(11.3 \%)$ and the pollen of shrubs and trees $(7.8 \%)$. Taking into account the known feeding habits of horses, these results suggest a meadowtype terrain, interspersed with bushes and shrubs, the leaves and shoots of which were consumed together with the herbiage.

The presence of pollens and spores from different ecological groups suggests that shortly before its death the horse had been ranging over different types of habitat-dry regions, mesophytic meadowland and damp riparian areas. It is noteworthy that the pollen grains of all types of plants, both monocotyledons and dicotyledons, were well matured, indicating that the horse perished in late summer. Since the Beresovka remains suggest an early summer dating, a slightly different ecological pattern between the two finds, irrespective of place or date, is to be expected, due to seasonal variation, and it will be interesting to see what differences further analysis may reveal.

Almost all the plants represented by the Selerikan find are still indigenous to northern Yakutia. The exceptions were a few grains of conifer pollen, notably Picea obovata. It is very possible, however, that these represent wind-blown pollen from the south that had settled on the grass and been ingested with it, rather than a part of the local flora.

\section{PHYSICAL METALLURGY}

\section{Order-disorder in Alloys}

from our Materials Science Correspondent

THE order $\rightleftarrows$ disorder transformation in alloys was the theme of an international symposium of metallurgists and physicists at Tübingen University from September 3-5. The proceedings, organised by Drs $\mathbf{H}$. Warlimont and W. Pitsch of the Max-Planck Gesellschaft, were largely concerned with the behaviour of solid solutions such as $\mathrm{Cu}_{3} \mathrm{Au}, \mathrm{Fe}_{3} \mathrm{Al}$, FeCo, $\mathrm{Ni}_{4} \mathrm{Mo}$, CuPt, which share the characteristic that at high temperatures the atomic distribution is wholly or almost wholly random but becomes ordered below a critical temperature. The distinguishing feature of the occasion was the emphasis placed on the statistical mechanics of the ordering process and also on its crystallographic and metallographic details; on the other hand the resultant changes in properties, especially mechanical ones, occupied a subordinate role.

The central concept in terms of which the ordering transformation has long been analysed is the interaction energy between first or second nearest neighbours, a variable which depends on the chemical identity of the neighbours. The interaction energies determine the extent of short-range ordering, the value of the critical temperature and the nature of the ordered "superlattice". Less directly, they determine ordering kinetics and also the form of the associated phase diagrams, that is, maps of the ordering behaviour of off-stoichiometric and two phase alloys. At the conference, substantial progress was reported on each of these fronts. Thus D. de Fontaine and H. Yamauchi (University of California, Los Angeles) were able to construct a novel theory of ordering kinetics in terms of pair-site probabilities (directly related to interaction energies), and a group led by W. Pitsch (MaxPlanck Institut für Eisenforschung, Düsseldorf) has made much progress in predicting phase diagrams in terms of interaction energies. C. M. van Baal
(Delft University of Technology) is also effectively active in this field. Physicists would also like to be able to move backwards to even greater generality by understanding neighbour interaction energies in terms of electronic characteristics of the constituent atoms and of the elastic and bond-theoretical characteristics of the crystals, but on this very difficult cluster of problems little progress was reported, and indeed none is on the horizon. One feature which is becoming clear, though, is that two-particle interactions are too simplistic an approach. For instance, P. Clapp (Ledgemont Laboratory of the Kennecott Copper Corporation, Lexington) showed convincingly that the peculiar ordered structure in CuPt can only be understood in terms of four-atom group interactions.

A generalised theory of superlattice types, by A. A. Smirnov (Ukranian Academy of Sciences, Kiev), was out-

\title{
More Suppressor T Cell Effects
}

Since the realization by at least some cellular immunologists that the sequence of cellular events following injection of an antigen should not be thought of as involving the linear differentiation of a single cell type, the fun has been fast and furious. Well-grown notions of six or seven years standing (the exact time depends upon the starting point) are those of $T$ lymphocytes (of thymic origin) and B lymphocytes (not of thymic origin). Inevitably various categories of $\mathrm{T}$ and $\mathrm{B}$ cells have been proposed; the macrophage has been dragged in and real enthusiasts have sought to embrace the mast cells, eosinophils, and sundry other cells. One of the latest and more entertaining manoeuvres has been the creation of the suppressor $\mathbf{T}$ cell and this is the subject of a communication by Okumura and Tada in Nature New Biology next Wednesday (October 10).

Adult donor mice were injected twice with either sheep or horse erythrocytes. Two weeks after the second injection they were killed and their spleens and thymuses were prepared, separately, for injection into recipient mice. These recipient mice were immunised once or twice with heavily dinitrophenylated horse red blood cells (DNP-HRBC) plus a pertussis adjuvant. The transfer of cells from the donor mice into the recipients was made at the time of either the first or the second immunising injection of DNP-HRBC. It was found that when the donor cells derived from an HRBC immunised mouse-that is, the carrier which is homologous to the carrier of DNP - the anti-DNP response was much suppressed, whereas cells from the heterologous carrier (SRBC) immunised donors had little or no such suppressive effect.
It did not matter whether the suppressive cells were culled from the thymus or the spleen of donor mice but in either instance their inhibitory effect could be abolished by incubation with anti- $\theta$ antiserum and complement before transfer. The conclusion from this last procedure is that the suppressive effect is exerted by $T$ cells. The authors remark that cells within the thymus are not normally regarded as susceptible of immunological activation, The specific suppressive effect of cells harvested from the thymuses of their donor mice does, however, suggest that in some circumstances the thymus should be thought of as directly involved in immunological responses.

The interest of experiments of this kind lies, first, in consideration of whether similar ' $\mathrm{T}$ ' cell suppressor effects occur in intact animals during the course of less controversial immunological responses and, second, in speculation as to the identity of the suppressive mechanism. No evidence is offered in the present experiments as to how the injected donor cells responded to their third contact with HRBC but other work suggests that $\mathrm{T}$-cell activation is a prerequisite of expression of suppressor function.

The present experiments of Okumura and Tada record a suppressive effect of $T$ cells on B cells which is measured by an anti-hapten response and although it is not explicitly stated there was presumably a suppression of the anti-carrier response. In a recent issue of Nature $(244,195 ; 1973)$ other suppressed targets of activated $\mathrm{T}$ cells were alluded to. These cells certainly seem equipped to perform both positive and negative regulation of immunological processes. 